\title{
Factors associated with refusal of radiotherapy among oral cancer patients
}

\author{
Agaku IT ${ }^{1, *}$ and Adisa $\mathrm{AO}^{2}$ \\ ${ }^{1}$ Center for Global Tobacco Control, Department of Society, Human Development \& Health, Harvard School of Public Health, Boston, MA, USA \\ ${ }^{2}$ Department of Oral Pathology, University College Hospital Ibadan, Nigeria
}

\begin{abstract}
Background: Surgery is commonly favored in the management of oral cancer but radiotherapy may be essential because of the size or location of the tumor. Refusal of radiotherapy by patients is an important issue, which must be taken into consideration during treatment planning. This study assessed prevalence and correlates of radiotherapy refusal among oral cancer patients. Methods: Data was analyzed for 47, 174 oral cancer cases in the Surveillance, Epidemiology and End Results (SEER) database during 1988-2008. Point estimates were calculated overall and by selected socio-demographic and clinical characteristics. A multivariate logistic regression model was fitted to determine predictors of radiotherapy refusal. Results: The overall prevalence of refusal of radiotherapy was $2.31 \%$. Factors associated with increased likelihood of refusal of radiotherapy included age $\geq 45$ years (adjusted odds ratio, aOR $=2.48$; $\mathrm{P}=0.031)$; gingival/floor of mouth tumors $(\mathrm{aOR}=1.32 ; P=0.010)$; receipt of surgery $(\mathrm{aOR}=1.21 ; P=0.04)$. Conversely, protective factors included being married (aOR=0.59; $P<0.001)$; non-Hispanic blacks (aOR=0.53; $P=0.001)$; involvement of paired structures $(\mathrm{aOR}=0.61 ; P<0.001)$ as well as multiple tumors $(\mathrm{aOR}=0.75 ; P=0.021)$. Sex was not a significant predictor on multivariate analysis. Conclusion: Prevalence of refusal of radiotherapy among oral cancer patients is relatively low and is significantly associated with age, marital status, as well as location, extent and severity of disease. Clinicians may anticipate patients likely to refuse radiotherapy and develop patient-tailored counseling considering the benefits and risks of proposed treatment. Final treatment decision must however take into consideration the wishes of the fully informed patient.
\end{abstract}

Keywords: oral cancer; malignancy; tumor; radiation; disparities; informed consent

\section{Introduction}

Tobacco use remains the single largest preventable cause of morbidity and mortality in the United States [1]. Despite declines in cigarette smoking, little change in the use of smokeless tobacco products has been observed in recent years [2]. In 2011, 3.2\% of persons aged 12 and older in the United States reported current use of smokeless tobacco products [2]. Smokeless tobacco contains tobacco-specific nitrosamines (TSNAs) which are the major carcinogens in tobacco [3]. The TSNAs N-nitrosonornicotine (NNN) and 4-(methylnitrosamino)-1-(3-pyridyl)-1-butanone (NNK) have been classified by the International Agency for Research on Cancer as group 1 carcinogens [3]. Even at low doses, NNK is a potent carcinogen and has been strongly associated with oral and esophageal cancer [3].

Surgery is commonly favored in the management of oral cancer but radiotherapy may be essential because of the size or location of the tumor. In late stage oral cancer, more aggressive treatment may be required [4]. Chemotherapy is often added to the treatment regimen in advanced tumors or tumors of certain sites in the hope of increasing control [4]. Radiotherapy could be used alone or as a planned combined modality with ablative tumor surgery, as well as in the treatment of elective neck volumes [4]. While better tumor control and reduced risk of local recurrence are seen with adjuvant or neoadjuvant radiotherapy for patients with well-defined risk factors, patients may still refuse radiotherapy administered alone or as a component of multimodal therapy [5]. Possible refusal of radiotherapy by patients is an important issue, which must be taken into consideration

*Corresponding author: Agaku IT, Center for Global Tobacco Control, Harvard School of Public Health, Boston, Massachusetts 02115, USA, Tel.: 770728 3220; E-Mail: iagaku@post.harvard.edu

Received 28 November 2012 Revised 7 March 2013 Accepted 15 March 2013 Published 22 March 2013

Citation: Agaku IT, Adisa AO (2013) Factors associated with refusal of radiotherapy among oral cancer patients. J Cancer Res Ther 1: 62-69. doi:10.14312/2052-4994.2013-10

Copyright: (C) 2013 Agaku IT, et al. This is an open-access article distributed under the terms of the Creative Commons Attribution License, which permits unrestricted use, distribution and reproduction in any medium, provided the original author and source are credited. 
by the clinician during treatment planning. Patients may object to radiotherapy for various personal, religious, socioeconomic, cultural, and health-related reasons [6]. It has been speculated that concerns about toxicities, limited social support, age-related changes regarding health and end of life issues or even the aggressiveness of physician recommendations may make patients refuse radiotherapy [6]. In line with patients' right to autonomy, it is the clinician's responsibility to provide the patient with the highest attainable standard of care within the boundaries of the patients' well informed medical decision. It is thus important for clinicians to know factors that might predict radiotherapy refusal in oral cancer patients.

Studies have reported the effect of refusal of radiotherapy in various cancers including breast, lung, head and neck, prostate, skin, gastrointestinal (esophagus, stomach, pancreas, colon, rectum/ recto-sigmoid), cervix, and uterus [6, 7]. However, relatively little is known about factors affecting refusal of radiotherapy in oral cancer patients. Thus, using the 1988-2008 Surveillance, Epidemiology, and End Results (SEER) data, this retrospective, population-based study explored predictors of radiotherapy refusal in patients diagnosed with oral cancer.

\section{Methods}

\section{Sources of data}

We used data from the SEER Program Research Data (1973-2008), National Cancer Institute, released April 2011, based on the November 2010 submission. We limited our analysis to cases reported during 1988-2008 [8].

We merged data from all 17 SEER registries-Atlanta, Connecticut, Detroit, Hawaii, Iowa, New Mexico, San Francisco-Oakland, Seattle-Puget Sound, Utah, San JoseMonterey, Los Angeles, Rural Georgia, Alaska Natives, Greater California, Kentucky, Louisiana, and New Jersey [8].

\section{Measures}

\section{Receipt of radiotherapy}

This was the primary outcome of interest and was dichotomized as "refused" vs. "administered". Cases in which patient or patient's guardian refused radiotherapy were categorized as "refused". We excluded cancer cases that were diagnosed at autopsy; those in which it was unknown if radiotherapy was recommended; as well as those in which it was not known if radiotherapy was performed even though recommended. The final analytical sample comprised of 47, 174 cases.

\section{Socio-demographic characteristics}

Socio-demographic characteristics assessed included: sex (male or female); marital status (never married; married (including common law); or separated, widowed or divorced); race/ethnicity (non-Hispanic white; non-
Hispanic black; non-Hispanic other race; or Hispanic); and age ( $\leq 34 ; 35-44 ; \geq 45$ years).

\section{Clinical characteristics}

Clinical characteristics assessed included histological grade of tumor (grades 1, 2, 3, 4); clinical stage of disease (in situ; localized; regional or distant (i.e., presence of metastasis)); laterality (involvement of paired or unpaired structures); site of tumor as defined using ICD-0-3 codes (lip (C000-C009); tongue/tonsils (C019-C029; C090C099); salivary glands (C079-C089); or gingiva/floor of mouth (C030-C069)); receipt of surgery (yes or no); and number of primary tumors (solitary tumor; 2 tumors; or $\geq 3$ tumors).

\section{Data analysis}

\section{Prevalence of refusal of radiotherapy}

The primary outcome of interest was refusal of radiotherapy among oral cancer patients. Point estimates were calculated overall and by sex, marital status, race/ ethnicity, age, histological grade of tumor, clinical stage of disease, laterality, site, receipt of surgery status, and site of primary tumor. Comparisons of prevalence of refusal of radiotherapy within groups were made using 95\% confidence intervals (CI); non-overlap of 95\%CIs indicated statistical significance.

\section{Bi and multivariate analysis}

X2 and Fisher's exact tests of independence were used as appropriate, to assess the unadjusted relationship between refusal of radiotherapy and sex, marital status, race/ethnicity, age, histological grade of tumor, clinical stage of disease, laterality, site, receipt of surgery status, and site of primary tumor. Statistical significance was tested at the $5 \%$ alpha level.

Selection of variables into the final multivariate model was based on statistical and clinical significance. Receipt of surgery status was included apriori based on clinical significance. A multivariate logistic regression model was fitted using the backward technique, adjusting for sex, marital status, race/ethnicity, age, histological grade of tumor, clinical stage of disease, laterality, site, receipt of surgery status, and site of primary tumor . Multivariate adjusted odds ratios and 95\%CIs were calculated for each variable. All analyses were performed with Stata version 12 (StataCorp 2011. Stata Statistical Software: College Station, TX).

\section{Results}

In total, $70.63 \%$ of patients were male and $29.37 \%$ were female. Over two-thirds (78.14\%) were non-Hispanic whites, $10.02 \%$ were non-Hispanic blacks, $5.32 \%$ were non-Hispanic other race while $6.52 \%$ were Hispanics. The majority of patients $(90.62 \%)$ were aged $\geq 45$ years. Patients with regional and metastatic disease made up $78.82 \%$ of the cohort, while patients with localized (20.71) 
and in situ $(0.47 \%)$ tumors made up the remainder. Most of the tumors where located on the lip (59.94\%), followed by the gingiva/floor of mouth $(23.84 \%)$, salivary glands (14.54\%) and tongue/tonsils (1.68\%). Over two-thirds of patients $(76.47 \%)$ had solitary tumors (Table 1 ).

The overall prevalence of refusal of radiotherapy was $2.31 \%$. On stratification by sex, prevalence of refusal of radiotherapy was lower among males $(2.09 \%$; $95 \% \mathrm{CI}$ : $1.94 \%-2.24 \%)$ compared to females $(2.84 \%$; $95 \% \mathrm{CI}$ : 2.57\%-3.12\%) (Table 1). However, after adjusting for all other covariates, there was no significant difference in refusal of radiotherapy between both sexes $(P=0.098)$ (Table 2).

Table 1 Patient socio-demographic and clinical characteristics of oral cancer patients, SEER, 1988-2008

\begin{tabular}{|c|c|c|c|c|}
\hline Characteristic & Number & Percentage, \% & $\begin{array}{l}\text { Proportion that refused } \\
\text { radiotherapy \% (95\% CI) }\end{array}$ & $\begin{array}{l}\text { Univariate test of independence } \\
\text { with refusal of radiotherapyt }\end{array}$ \\
\hline Overall & 47,174 & 100 & $2.31(2.18-2.45)$ & $n / a$ \\
\hline \multicolumn{5}{|l|}{ Socio-demographic factors } \\
\hline \multicolumn{5}{|l|}{ Sex } \\
\hline Male & 33,318 & 70.63 & $2.09(1.94-2.24)$ & $<0.001$ \\
\hline Female & 13,856 & 29.37 & $2.84(2.57-3.12)$ & \\
\hline \multicolumn{5}{|l|}{ Marital status } \\
\hline Never married & 7,719 & 17.01 & $2.55(2.20-2.90)$ & $<0.001$ \\
\hline Married (including common law) & 25,669 & 56.57 & $1.74(1.58-1.90)$ & \\
\hline Separated, widowed or divorced & 11,989 & 26.42 & $3.22(2.90-3.54)$ & \\
\hline \multicolumn{5}{|l|}{ Race/ethnicity } \\
\hline Non-Hispanic white & 36,765 & 78.14 & $2.32(2.17-2.47)$ & $<0.001$ \\
\hline Non-Hispanic black & 4,716 & 10.02 & $1.84(1.46-2.23)$ & \\
\hline Non-Hispanic other race & 2,501 & 5.32 & $3.28(2.58-3.98)$ & \\
\hline Hispanic & 3,070 & 6.52 & 1.89 (1.41-2.37) & \\
\hline \multicolumn{5}{|l|}{ Age, years } \\
\hline$\leq 34$ & 1,102 & 2.34 & $1.63(0.88-2.38)$ & $<0.001$ \\
\hline $35-44$ & 3,321 & 7.04 & $1.39(0.99-1.78)$ & \\
\hline$\geq 45$ & 42,750 & 90.62 & $2.40(2.25-2.55)$ & \\
\hline \multicolumn{5}{|l|}{$\begin{array}{l}\text { Clinical variables } \\
\text { Histological grade }\end{array}$} \\
\hline Grade 1 & 4,139 & 11.09 & $2.71(2.21-3.20)$ & $<0.001$ \\
\hline Grade 2 & 17,334 & 46.44 & $2.40(2.17-2.63)$ & \\
\hline Grade 3 & 14,275 & 38.25 & 1.77 (1.56-1.99) & \\
\hline Grade 4 & 1,576 & 4.22 & $2.09(1.39-2.80)$ & \\
\hline \multicolumn{5}{|l|}{ Clinical stage } \\
\hline In situ & 213 & 0.47 & $3.76(1.20-6.32)$ & $<0.001$ \\
\hline Localized & 9,373 & 20.71 & $2.89(2.55-3.23)$ & \\
\hline Regional & 29,562 & 65.33 & $1.89(1.73-2.04)$ & \\
\hline Distant (metastasis) & 6,105 & 13.49 & $2.72(2.31-3.13)$ & \\
\hline
\end{tabular}




\begin{tabular}{|c|c|c|c|c|}
\hline Unpaired structures & 27,481 & 58.25 & $2.76(2.57-2.96)$ & $<0.001$ \\
\hline Paired structures & 19,693 & 41.75 & $1.68(1.50-1.86)$ & \\
\hline \multicolumn{5}{|l|}{ Site } \\
\hline Lip & 28,277 & 59.94 & $2.03(1.87-2.20)$ & $<0.001$ \\
\hline Tongue/tonsils & 791 & 1.68 & $2.15(1.14-3.16)$ & \\
\hline Gingiva/floor of mouth & 11,245 & 23.84 & $3.22(2.89-3.55)$ & \\
\hline Salivary gland & 6,861 & 14.54 & $1.98(1.65-2.31)$ & \\
\hline \multicolumn{5}{|l|}{ Surgery } \\
\hline None & 15,829 & 46.27 & $2.27(2.04-2.51)$ & 0.828 \\
\hline Received & 18,378 & 53.73 & $2.31(2.10-2.53)$ & \\
\hline \multicolumn{5}{|c|}{ Number of primary tumors } \\
\hline Solitary tumor & 36,072 & 76.47 & $2.45(2.29-2.61)$ & $<0.001$ \\
\hline 2 tumors & 8,902 & 18.87 & $1.81(1.53-2.09)$ & \\
\hline$\geq 3$ tumors & 2,200 & 4.66 & $2.09(1.49-2.69)$ & \\
\hline
\end{tabular}

Abbreviations: $\mathrm{CI}=$ confidence interval; $\mathrm{n} / \mathrm{a}=$ not applicable; SEER=Surveillance, Epidemiology and End Results database $\dagger \mathrm{X} 2$ test of independence was used for sex, laterality and surgery receipt status while Fisher's exact test was used for all other variables

Table 2 Predictors of refusal of radiotherapy among oral cancer patients, SEER, 1988-2008

\begin{tabular}{|c|c|c|c|c|}
\hline Characteristic & $\begin{array}{c}\text { Adjusted odds ratio } \\
(95 \% \mathrm{CI})\end{array}$ & P-value & $\begin{array}{c}\text { Crude odds ratio } \\
\text { (95\% CI) }\end{array}$ & P-value \\
\hline \multicolumn{5}{|l|}{$\operatorname{Sex}$} \\
\hline \multicolumn{5}{|l|}{ Male } \\
\hline Female & $1.17(0.97-1.41)$ & 0.098 & $1.37(1.21-1.55)$ & $<0.001$ \\
\hline \multicolumn{5}{|l|}{ Marital status } \\
\hline \multicolumn{5}{|l|}{ Never married $\neq$} \\
\hline Married (including common law) & $0.59(0.46-0.74)$ & $<0.001$ & $0.68(0.57-0.8)$ & $<0.001$ \\
\hline Separated, widowed or divorced & $0.97(0.76-1.24)$ & 0.815 & $1.27(1.07-1.51)$ & 0.007 \\
\hline \multicolumn{5}{|l|}{ Race/ethnicity } \\
\hline \multicolumn{5}{|l|}{ Non-Hispanic whiteł } \\
\hline Non-Hispanic black & $0.53(0.37-0.77)$ & 0.001 & $0.79(0.63-0.99)$ & 0.039 \\
\hline Non-Hispanic other race & $1.13(0.79-1.61)$ & 0.511 & $1.43(1.13-1.80)$ & 0.002 \\
\hline Hispanic & $0.88(0.62-1.26)$ & 0.492 & $0.81(0.62-1.06)$ & 0.126 \\
\hline \multicolumn{5}{|l|}{ Age, years } \\
\hline \multicolumn{5}{|l|}{$\leq 34 \neq$} \\
\hline $35-44$ & $1.53(0.62-3.8)$ & 0.355 & $0.85(0.49-1.46)$ & 0.55 \\
\hline$\geq 45$ & $2.48(1.09-5.65)$ & 0.031 & $1.48(0.93-2.37)$ & 0.101 \\
\hline
\end{tabular}




\author{
Clinical variables \\ Histological grade \\ Grade 1キ
}

Grade 2

Grade 3

Grade 4

Clinical stage

In situ‡

Localized

Regional

Distant (metastasis)

Laterality

Unpaired structuresł

Paired structures

Site

Lipł

Tongue/tonsils

Gingiva/floor of mouth

Salivary gland

Surgery

Noneł

Received

$1.21(1.01-1.46)$

0.04

$1.02(0.88-1.17)$

0.814
$0.45(0.06-3.47)$

0.25 (0.03-1.96)

0.37 (0.05-2.88)

$0.61(0.48-0.77)$

$0.8(0.39-1.64)$

1.32 (1.07-1.62)

$1.13(0.80-1.60)$
0.82

$<0.001$

0.787

$\begin{array}{ll}0.49(0.24-1.00) & 0.051 \\ 0.72(0.35-1.48) & 0.366\end{array}$

$0.60(0.53-0.69)$

$<0.001$
0.97 (0.81-1.18)

$1.60(1.40-1.83)$
0.254

0.19

.366
Number of primary tumors

Solitary tumor‡

2 tumors

$\geq 3$ tumors 
2.55\%) (Table 1). After adjusting for all other covariates, the odds of refusing radiotherapy were 2.48 times higher among patients aged $\geq 45$ years compared to those aged $\leq 34$ years $(P=0.031)$. There was however no significant difference between patients aged 35-44 years and those aged $\leq 34$ years $(P=0.355)$ (Table 2$)$. By histological grade of tumor, prevalence of refusal of radiotherapy was highest among patients with grade 1 tumors $(2.71 \%$; $95 \%$ CI: $2.21 \%-3.20 \%$ ) and lowest among those with grade 3 tumors (1.77\%; 95\% CI: 1.56\%-1.99\%) (Table 1).Similarly, by clinical stage, patients with regional disease had the lowest prevalence of refusal of radiotherapy $(1.89 \%$; $95 \%$ CI: $1.73 \%-2.04 \%$ ) while those with in situ disease had the highest prevalence of refusal of radiotherapy $(3.76 \%$; $95 \%$ CI: $1.20 \%-6.32 \%)$. These differences by grade and stage were however not statistically significant after adjusting for all other covariates (Table 2).

Prevalence of refusal of radiotherapy was significantly lower among patients with tumors involving paired structures compared to patients with involvement of unpaired structures (1.68\%; 95\%CI: $1.50 \%-1.86 \%$ vs. 2.76\%; 95\%CI: 2.57\%-2.96\%, respectively) (Table 1). On multivariate analysis, the odds of refusing radiotherapy were 39\% lower among patients with affectation of paired structures compared to those with unpaired structures $(\mathrm{aOR}=0.61 ; \quad P<0.001)$ (Table 2). By site of primary tumor, patients with gingival/floor-of-mouth lesions had the highest prevalence of refusal of radiotherapy (3.22\%; 95\% CI: $2.89 \%-3.55 \%$ ) whereas those with salivary gland lesions had the lowest prevalence $(1.98 \%$; $95 \% \mathrm{CI}$ : 1.65\%-2.31\%). After adjusting for all other covariates, the odds of refusing radiotherapy were 1.32 times higher among patients with tumors involving the gingiva/floorof-mouth compared to those with lip lesions $(P=0.010)$. However, there were no significant differences between patients with tumors of the tongue/tonsils, or salivary gland when compared to those with lip lesions.

The prevalence of refusal of radiotherapy was $2.27 \%$ (95\%CI: $2.04 \%-2.51 \%$ ) among patients that did not receive surgery and $2.31 \%$ (95\%CI: $2.10 \%-2.53 \%$ ) among surgery recipients (Table 1). After adjusting for all covariates, receipt of surgery emerged as a significant predictor of refusal of radiotherapy; surgery recipients were 1.21 times more likely to refuse radiotherapy compared to those who did not receive surgery $(P=0.04)$ (Table 2). Also, prevalence of refusal of radiotherapy was highest among those with solitary tumors $(2.45 \%$; $95 \%$ CI: $2.29 \%-2.61 \%$ ) and lowest among those with two primary tumors (1.81\%; 95\% CI: 1.53\%-2.09\%). After adjusting for all covariates, patients with two primary tumors had 25\% lower odds of refusing radiotherapy compared to those with a solitary tumor $(\mathrm{aOR}=0.75$; $P=0.021$ ). However, the difference between patients with $\geq 3$ tumors and those with solitary tumors did not attain statistical significance on multivariate analysis (aOR=0.80; $P=0.338)$ (Table 2).

\section{Discussion}

This study showed that approximately $2.31 \%$ of all oral cancer patients refused radiotherapy. This is similar to previous research which reported that $1.6 \%$ of patients with head and neck cancers refused radiotherapy [6]. These two studies report relatively lower rates of refusal for radiotherapy compared to those who refused radiotherapy for prostate $(7.6 \%)$ or colon (8.4\%) cancers [6]. Patients with head and neck cancer (including oral cancer) have specific requirements that are beyond the needs of patients diagnosed with other types of cancers. Oral cancer patients encounter difficulties in eating, chewing, drinking, breathing, speaking, as well as aesthetic challenges [9]. Many of these patients cannot conceal the noticeable alterations in their appearance. Head and neck cancers have thus being described as being more "emotionally traumatic" than any other type of cancer [9]. Also, the burden of head and neck cancer is often manifested in psychosocial dysfunction, which has a negative impact on quality of life [10]. These difficulties may tend to make patients with head and neck cancer comply with recommended therapy more than patients with cancers at other sites.

The American Hospital Association and the U.S. Supreme Court have each delineated certain rights to which patients who have the competence and mental capacity to make decisions for themselves are entitled, including the right to refuse treatment [11]. This right may be particularly important to geriatric patients, because a given treatment may have differential risks or benefits based on age of patient, due to differences in immune-competence, general health condition and other co-morbidities and disabilities, degree of physical activity, ease of access to healthcare and social support, as well as other physical, attitudinal, social and behavioral characteristics. In our study, refusal of radiotherapy was higher among older patients, which is consistent with earlier research [6]. Elderly patients may be less willing or able to tolerate oral cancer radiotherapy because of frailty, and fear of compromise to quality of life from complications of radiotherapy such as dysphagia, soft tissue or bone necrosis, and radiation-induced neuropathy [12].

We found that married persons were less likely to refuse radiotherapy, compared to those who were never married. The social support provided by marriage has been reported to benefit cancer patients as it enhances compliance with therapy and improves patients' immune system via psychosomatic mechanisms [13]. Interestingly, there was no significant difference between separated, widowed or divorced patients, and those who were never married, thereby underscoring the possible role of social capital provided in the marriage setting in treatment compliance [13].

The lower rates of refusal of radiotherapy among nonHispanic blacks compared to non-Hispanic whites may be multi-factorial and related to differences in stage of 
cancer diagnosis, access to health care counseling and treatment alternatives $[14,15]$. Research has shown that blacks are more likely to be diagnosed with late stage cancer compared to whites due to lower access to cancer screening [15]. Also, blacks have been shown to be less likely to receive cancer directed surgery compared to whites [14]. Taken together, these factors may suggest that at time of presentation and diagnosis, blacks may be more likely to be offered or to benefit from radiotherapy due to regional or distant spread of disease. This is consistent with studies that have shown higher rates of radiotherapy among blacks with head and neck cancer compared to whites [15]. Sustained and enhanced efforts are needed to reduce racial disparities in oral cancer screening and access to care.

Patients with multiple tumors as well as those with tumors originating from paired structures were less likely to refuse radiotherapy. The number of tumors or the involvement of paired structures may be an index of severity of disease or may suggest a worse prognosis. A study assessing survival in breast cancer patients found that women with synchronous bilateral breast cancer and those with metachronous bilateral breast cancers diagnosed within two years of the original primary had worse survival compared to those with unilateral breast cancer [16]. Thus, it is likely that patients with multiple cancers may more readily comprehend the magnitude or severity of the disease. Multiple tumors may indicate multiple surgical sites or more extensive/complex ablative surgeries, and thus patients may be more willing to comply with a less invasive therapy like radiotherapy in addition with minimal surgery to prevent psychological disturbances, deformity and loss of function in the maxillofacial region $[17,18]$. In addition, this could also reflect enhanced counseling by clinicians for patients with higher risk of recurrence.

Site of tumor was a significant predictor of refusal of radiotherapy, with patients with lip lesions being more amenable to radiotherapy compared to those with gingival/floor-of-mouth lesions. This may be due to aesthetic considerations as lip lesions may be more obvious and emotionally distressing to the patient compared to floor of mouth or gingival lesions, and may thus contribute to better compliance with proposed therapy [9]. Involvement of vital structures such as the tongue may equally result in high acceptance of radiotherapy. Our study found no significant difference in refusal of radiotherapy among patients with tongue lesions compared to those with lip lesions. In addition, we found that patients that underwent surgery were more likely to refuse radiotherapy compared to non-recipients of surgery. A possible explanation could be that good tumor control was achieved through ablative surgery. However, prior research has indicated that patients may decline multimodal therapy because of co-morbidities, and concerns about toxicity [19].

\section{Clinical implications}

This study highlights some correlates of radiotherapy refusal among oral cancer patients, which could be used by physicians to anticipate and prepare for objections about radiotherapy in patients with oral cancer. These factors include socio-demographic and clinical predictors and may also include a combination of these. Based on clinical assessment of possible benefits and risks of proposed treatment, clinicians may provide targeted counseling to patients who refuse radiotherapy. However, the final modality of treatment must be in accord with patient's well informed wishes.

\section{Strengths and limitations}

We used data from all SEER registries, thus the findings are generalizable. A limitation of the study was the inability to adjust for socioeconomic status of patients as this data was not available in SEER. Differences in socioeconomic status may impact health seeking behavior, risk-modifying behavior (e.g., smoking), and access to health insurance and high quality medical care. The findings from this study should therefore be interpreted in the light of this limitation.

\section{Conclusion}

The overall prevalence of refusal of radiotherapy was $2.31 \%$. Refusal of radiation was significantly associated with age, marital status, race/ethnicity, laterality, site and number of tumors as well as receipt of surgery status. Sex was not a significant predictor of refusal of radiation therapy, after adjusting for all other covariates. Clinicians may anticipate patients likely to refuse radiotherapy and develop patient-tailored counseling considering the benefits and risks of proposed treatment. Final treatment decision must however take into consideration the wishes of the fully informed patient.

\section{Conflict of interest}

The authors wish to express that they have no conflict of interest.

\section{References}

[1] Preventing tobacco use among youth and young adults: a report of the surgeon general (2012) Atlanta, GA: U.S Department of Health and Human Services, Centers for Disease Control and Prevention, National Center for Chronic Disease Prevention and Health Promotion, Office on Smoking and Health.

[2] Substance abuse and mental health services administration, results from the 2011 national survey on drug use and health: summary of national findings, NSDUH series H-44, HHS Publication No. (SMA) 12-4713. Rockville, MD: Substance Abuse and Mental Health Services Administration, 2012.

[3] Hatsukami DK, Lemmonds C, Zhang Y, Murphy SE, Le C, et al. (2004) Evaluation of carcinogen exposure in people who used "reduced exposure" tobacco products. J Natl Cancer Inst 96:844-852.

[4] Vokes EE, Athanasiadis I (1996) Chemotherapy of squamous cell carcinoma of head and neck: the future is now. Ann Oncol 7:15-29.

[5] Malone JP, Stephens JA, Grecula JC, Rhoades CA, Ghaheri BA, et al. (2004) Disease control, survival, and functional outcome after multimodal treatment for advanced-stage tongue base cancer. Head Neck 26:561-572. 
[6] Hamidi M, Moody JS, Kozak KR (2010) Refusal of radiation therapy and its associated impact on survival. Am J Clin Oncol 33: 629632.

[7] Ma AM, Barone J, Wallis AE, Wu NJ, Garcia LB, et al. (2008) Noncompliance with adjuvant radiation, chemotherapy, or hormonal therapy in breast cancer patients. Am J Surg 196:500504.

[8] Surveillance, Epidemiology, and End Results (SEER) Program research data (1973-2008), National Cancer Institute, DCCPS, surveillance research program, cancer statistics branch, released April 2011, based on the November 2010 submission.

[9] Koster ME, Bergsma J (1990) Problems and coping behaviour of facial cancer patients. Soc Sci Med 30:569-578.

[10] Semple C (2001) The role of the CNS in head and neck oncology. Nurs Stand 15:39-42.

[11] Plawecki LH, Amrhein DW (2009) When "no" means no: elderly patients' right to refuse treatment. J Gerontol Nurs 35:16-18.

[12] Hermans R (2008) Posttreatment imaging in head and neck cancer. Eur J Radiol 66:501-511.

[13] Ell K, Nishimoto R, Mediansky L, Mantell J, Hamovitch M (1992) Social relations, social support and survival among patients with cancer. J Psychosom Res 36:531-541.

[14] Shavers VL, Harlan LC, Winn D, Davis WW (2003) Racial/ethnic patterns of care for cancers of the oral cavity, pharynx, larynx, sinuses, and salivary glands. Cancer Metastasis Rev 22:25-38.

[15] Tomar SL, Loree M, Logan H (2004) Racial differences in oral and pharyngeal cancer treatment and survival in Florida. Cancer Causes Control 15:601-609.

[16] Kollias J, Ellis IO, Elston CW, Blamey RW (2001) Prognostic significance of synchronous and metachronous bilateral breast cancer. World J Surg 25:1117-1124.

[17] Beumer J, Curtis TA, Marunick MT (1996) Maxillofacial rehabilitation: prosthetic and surgical consideration. St Louis: Medico Dental Media International p 18.

[18] Stanley J (2000) Oral complications of radiotherapy and their management: a review. Detroit Dent Bull 69: 7-8.

[19] El Shayeb M, Scarfe A, Yasui Y, Winget M (2012) Reasons physicians do not recommend and patients refuse adjuvant chemotherapy for stage III colon cancer: a population based chart review. BMC Res Notes 5:269. 